\title{
Atuação da enfermagem na assistência ao paciente em cuidados paliativos: uma revisão integrativa
}

\begin{abstract}
RESUMO | Objetivo: Analisar as evidências científicas disponíveis na literatura sobre a atuação da enfermagem na assistência ao paciente em cuidados paliativos. Método: Trata-se de uma revisão integrativa. Realizou-se a busca por artigos com delimitação nos últimos 5 anos; nos idiomas português, inglês e espanhol; disponíveis na íntegra. Nas plataformas de dados: BDENF, DOAJ, LILACS, PMC, SCOPUS e Web of Science. Resultados: Os dados foram organizados e apresentados em figuras e tabelas. Dos 125 estudos encontrados, 25 estava disponível na BDENF, 46 na DOAJ, 24 na LILACS, 26 na PMC, 0 na SCOPUS, e 4 na Web of Science; contudo, após a leitura permaneceram apenas os que atendiam aos critérios para inclusão e exclusão descritos na metodologia, 6 estudos. Conclusão: Este estudo proporcionou a identificação de algumas dificuldades e das características associadas a atuação da enfermagem na assistência ao paciente em cuidados paliativos; vinculado ao déficit de conhecimento e falta de incentivo.
\end{abstract}

Descritores: Enfermagem; Cuidados Paliativos; Humanização da Assistência

\begin{abstract}
Objective: To analyze the scientific evidence available in the literature on the role of nursing in patient care in palliative care. Method: This is an integrative review. The search for articles was carried out; with delimitation in the last 5 years; in Portuguese, English and Spanish; available in full. On data platforms: BDENF, DOAJ, LILACS, PMC, SCOPUS and Web of Science. Results: Data were organized and presented in figures and tables. Of the 125 studies found, 25 were available from BDENF, 46 from DOA, 24 from LILACS, 26 from PMC, 0 from SCOPUS, and 4 from Web of Science; however, after reading, only those who met the criteria for inclusion and exclusion described in the methodology remained, 6 studies. Conclusion: This study provided the identification of some difficulties and characteristics associated with the role of nursing in patient care in palliative care; linked to the lack of knowledge and lack of incentive.
\end{abstract}

Keywords: Nursing; Palliative Care; Humanization of Assistance

RESUMEN | Objetivo: Analizar la evidencia científica disponible en la literatura sobre el papel de la enfermería en el cuidado del paciente en cuidados paliativos. Método: Esta es una revisión integradora. Se realizó la búsqueda de artículos; con delimitación en los últimos 5 años; en portugués, inglés y español; disponible en su totalidad. En plataformas de datos: BDENF, DOAJ, LILACS, PMC, SCOPUS y Web of Science. Resultados: Los datos se organizaron y presentaron en figuras y tablas. De los 125 estudios encontrados, 25 estaban disponibles en BDENF, 46 en DOAJ, 24 en LILACS, 26 en PMC, 0 en SCOPUS y 4 en Web of Science; sin embargo, después de la lectura, solo quedaron aquellos que cumplieron con los criterios de inclusión y exclusión descritos en la metodología, 6 estudios. Conclusión: Este estudio permitió identificar algunas dificultades y características asociadas al papel de la enfermería en el cuidado del paciente en cuidados paliativos; vinculado a la falta de conocimiento y falta de incentivos.

Palabras claves: Enfermería; Cuidados Paliativos; Humanización de la Atención

\section{Maria de Fátima Silva Nascimento}

Discente do Curso de Bacharel em Enfermagem pelo Centro Universitário Maurício de Nassau (UNINASSAU). Recife, Pernambuco (PE), Brasil.

ORCID: 0000-0002-3991-8527.

\section{Liniker Scolfild Rodrigues da Silva}

Especialista em Enfermagem Obstétrica na modalidade Residência pela Faculdade de Enfermagem Nossa Senhora das Graças/Universidade de Pernambuco (FENSG/ UPE). Sanitarista, Especialista em Saúde Coletiva na modalidade Residência pela Faculdade de Ciências Médicas (FCM)/ UPE. Recife, Pernambuco (PE), Brasil. ORCID: 0000-0003-3710-851X

\section{Luzeni Maria Soares}

Especialista em Urgência e Emergência com ênfase em Atendimento Pré-Hospitalar (APH) e Especialista em Unidade de Terapia Intensiva (UTI) pela Faculdade Metropolitana de Ciências e Tecnologia (FAMEC). Especialista em Saúde Coletiva com ênfase na Organização e a Administração da Saúde Pública - Sanitarista pela Faculdade Integrada de Gestão e Meio Ambiente (FACIGMA). Recife, Pernambuco (PE), Brasil

ORCID: 0000-0003-3148-1815

\section{Alyne Silva dos Santos}

Enfermeira (egressa) pelo Instituto Pernambucano de Ensino Superior (IPESU). Recife, Pernambuco (PE), Brasil.

ORCID: 0000-0003-3131-3379

\section{Rayssa Sydnara Angelo Tavares}

Enfermeira (egressa) pelo Instituto Pernambucano de Ensino Superior (IPESU). Recife, Pernambuco (PE), Brasil. ORCID: 0000-0001-7179-0116

\section{Daniela Vieira da Silva}

Enfermeira (egressa) pelo Instituto Pernambucano de Ensino Superior (IPESU). Recife, Pernambuco (PE), Brasil. ORCID: 0000-0003-3367-2260

Recebido em: 21/07/2021 Aprovado em: 09/08/2021 


\section{INTRODUÇÃO}

A ntes mesmo da Organização Mundial da Saúde (OMS) definir os cuidados paliativos como uma área de cuidado onde a qualidade de vida de um paciente com doença diagnosticada era priorizada além da possibilidade de tratamento, independente do tempo que o paciente restava para diagnosticar a doença. Comprometida em cuidar dos pacientes, Florence percebe que é mais do que apenas tomar remédios ${ }^{(1)}$.

Os cuidados paliativos baseiam-se no seguinte princípio: reafirmar o sentido da vida, mas lembrar também que a morte é um processo totalmente natural que define o ciclo vital do cuidado e não tem por objetivo acelerar ou prolongar desproporcionalmente o tempo da morte. Ação (persistência do tratamento); aliviar dores e outros sintomas, aumentar aspectos psicológicos e espirituais, entender sua importância nas estratégias de enfermagem, e não se esquecer da importância de apoiar os familiares para que possam enfrentar este momento e deixá-los preparados para o momento triste ${ }^{(2,3)}$.

São indicados para pacientes de todas as idades, sejam adultos ou crianças, que sofrem de qualquer tipo de doença, mas ainda não alcançaram um tratamento que leve à cronicidade dessa patologia. A dor e o sofrimento desses pacientes são acompanhados de sintomas psicológicos, sociais e espirituais. Apoio extensivo à família ${ }^{(4,5)}$.

Diagnosticar doenças que afetam a saúde e a continuidade da vida levanta várias questões sobre os melhores cuidados. Os cuidados paliativos são vistos como uma linha de cuidado cujo principal objetivo é manter a qualidade de vida e proporcionar conforto à medida que a doença progride. Esse tipo de atendimento exige uma equipe treinada que possa levar em consideração todas as dimensões do paciente ${ }^{(5)}$.

Embora os cuidados paliativos tenham progredido, é preciso enfatizar que os não estão capacitados a cuidar desses pacientes, e os sentimentos de depressão, impotência e ignorância continuam a dificultar o andamento do cuidado. Se os profissionais responsáveis por esses pacientes passarem por aprendizagem e treinamento ao longo da vida, acumularem conhecimentos teóricos e experiência prática e, assim, darem uma contribuição significativa para a melhoria das condições de vida dos pacientes nesse processo, será diferente.

Por este motivo, este estudo tem como objetivo analisar as evidências

\section{Diagnosticar doenças que afetam a saúde e a continuidade da vida levanta várias questões sobre os melhores cuidados.}

científicas disponíveis na literatura sobre a atuação da enfermagem na assistência ao paciente em cuidados paliativos.

\section{MÉTODO}

Trata-se de um estudo bibliográfico, descritivo do tipo revisão integrativa, com abordagem qualitativa que oferece oportunidades para analisar a literatura científica e compreender amplamente os tópicos de pesquisa, contribuindo assim para as práticas de atendimento ao paciente com base no conhecimen- to científico ${ }^{(6)}$.

Determinou-se o cumprimento das seguintes etapas: (1) elaboração da questão norteadora e objetivo do estudo; (2) definição de critérios de inclusão e exclusão das produções científicas; (3) busca de estudos científicos nas bases de dados e bibliotecas virtuais; (4) análise e categorização das produções encontradas; (5) resultados e discussão dos achados ${ }^{(7)}$.

Para o levantamento da questão norteadora, utilizou-se a estratégia PICo, uma metodologia que auxilia na construção de uma pergunta de pesquisa e busca de evidências para uma pesquisa não-clínica, onde $\mathrm{P}=$ População/ Paciente; I = Interesse; e Co = Contexto (P: Paciente em Cuidados Paliativos; I: Assistência de Enfermagem; Co: Assistência de Qualidade). Desta forma, definiu-se a seguinte questão norteadora da pesquisa: "Como a enfermagem atua na assistência ao paciente em cuidados paliativos?".

Para seleção dos artigos, utilizaram-se como critérios de inclusão: artigo original, disponíveis na íntegra, publicado nos últimos 5 anos (2015-2020) em português, inglês ou espanhol, que respondessem ao objetivo do estudo. Foram excluídas as literaturas cinzas, bem como publicações repetidas de estudos em mais de uma base de dados e os artigos que não responderam à questão norteadora do estudo e que possibilitasse o acesso pelo Virtual Private Network (VPN) da Universidade de Pernambuco (UPE). Justifica-se a delimitação temporal nos últimos 5 anos visando o levantamento de artigos recentes.

O levantamento dos dados ocorreu durante o mês de maio e junho de 2021 nas seguintes Bases de Dados: Base de Dados de Enfermagem (BDENF); Directory of Open Access Journals (DOAJ); Literatura Latino-Americana e do Caribe em Ciências da Saúde (LILACS); PubMed Central (PMC), SCOPUS, e na Web of Science. 
Quadro 1 - Estratégia de busca por base de dados. Recife, Pernambuco (PE), Brasil, 2021.

\begin{tabular}{|c|c|c|c|}
\hline Bases de dados & Termos da Busca & Resultados & Selecionados \\
\hline BDENF & $\begin{array}{l}\text { Nursing AND Palliative Care AND Humani- } \\
\text { zation of Assistance }\end{array}$ & 25 & 2 \\
\hline DOAJ & $\begin{array}{l}\text { Nursing AND Palliative Care OR Humaniza- } \\
\text { tion of Assistance }\end{array}$ & 46 & 2 \\
\hline LILACS & $\begin{array}{l}\text { Nursing AND Palliative Care AND Humani- } \\
\text { zation of Assistance }\end{array}$ & 24 & 1 \\
\hline PMC & $\begin{array}{l}\text { Nursing AND Palliative Care AND Humani- } \\
\text { zation of Assistance }\end{array}$ & 26 & 0 \\
\hline SCOPUS & $\begin{array}{l}\text { Nursing AND Palliative Care AND Humani- } \\
\text { zation of Assistance }\end{array}$ & 0 & 0 \\
\hline Web of Science & $\begin{array}{l}\text { Nursing AND Palliative Care AND Humani- } \\
\text { zation of Assistance }\end{array}$ & 4 & 1 \\
\hline Total & & 125 & 6 \\
\hline
\end{tabular}

Fonte: Dados da pesquisa, 2021. eliminaram-se por meio da leitura de títulos e resumos, estudos duplicados. Destes pré-selecionados, realizou-se leitura na íntegra, a fim de verificar os que atendem à questão norteadora e aos critérios de inclusão/exclusão. Construiu-se então a amostra final com estudos pertinentes aos critérios pré-estabelecidos (Figura 1).

Após a leitura dos artigos selecionados os estudos foram categorizados, classificando o conhecimento produzido em níveis de evidência de acordo com Melnyk \& Fineout-Overholt (9) : nível I, as evidências são relacionadas à revisão sistemática ou metanálise de ensaios clínicos randomizados controlados ou provenientes de diretrizes

Figura 1 - Fluxograma do processo de seleção dos estudos primários adaptado do PRISMA. Recife, Pernambuco (PE), Brasil, 2021.
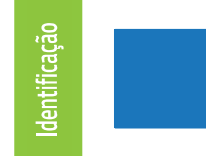

Publicações identificadas nas bases de dados $(n=125)$ BDENF - 25; DOAJ - 46; LILACS - 24; PMC - 26; SCOPUS -0 ; Web of Science -4 .
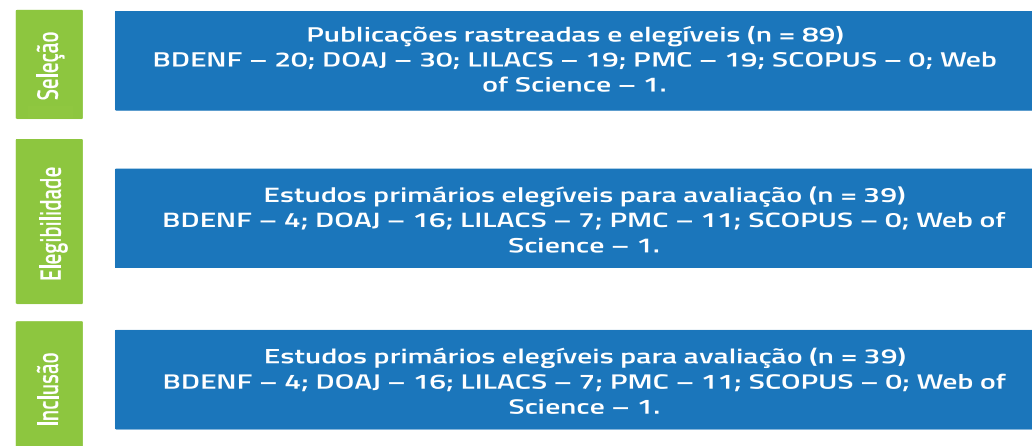

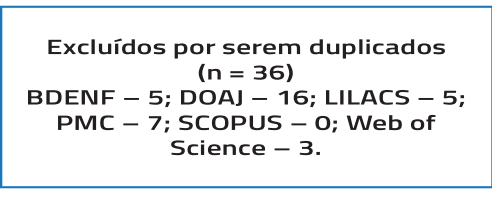

Excluídos após leitura de títulos e resumos por não atenderem aos Critérios de inclusão $(n=50)$
BDENF - 16; DOAJ - 14; LILACS - 12; PMC - 8; SCOPUS -0 ; Web of Science -0 .

Excluídos por não responderem à questão da pesquisa $(n=33)$ BDENF - 2; DOAJ - 14; LILACS - 6 PMC - 11; SCOPUS - O; Web of Science -0 .
Fonte: Recife, Pernambuco (PE), Brasil, 2021.

Buscaram-se os artigos indexados a partir dos Descritores em Ciências da Saúde (DeCS): "Enfermagem", "Cuidados Paliativos", "Humanização da Assistência". Utilizaram-se os respectivos termos provenientes do Medical Subject Headings (MeSH): "Nursing", "Palliative Care", "Humanization of Assistance". A operacionalização e a estratégia de busca se deram a partir da combinação com operador booleano AND e OR, efetuando a busca conjunta e individualmente para que possíveis diferenças fossem corrigidas (Quadro $1)$.

A seleção dos estudos baseou-se no Preferred Reporting Items for Systematic Review and Meta-Analyse (PRISMA) (8) com o objetivo de auxiliar no desenvolvimento de artigos. A princípio clínicas baseadas em revisões sistemáticas de ensaios clínicos randomizados controlados; no nível II, evidências derivadas de no mínimo um ensaio clínico randomizado controlado bem delineado; no nível III, evidências de ensaios clínicos bem delineados sem randomização; no nível IV, evidências advindas de estudos de coorte e de caso-controle bem delineados; no nível V, evidências 
provenientes de revisão sistemática de estudos descritivos e qualitativos; no nível VI, evidências derivadas de um único estudo descritivo ou qualitativo; e no nível VII, evidências derivadas de opinião de autoridades e/ou relatório de comitês de especialistas.

Obteve-se a sumarização das informações do corpus por meio de um instrumento: identificação do artigo original; autoria do artigo; ano de publicação; país; características metodológicas do estudo; e amostra do estudo.
Foi realizada leitura analítica dos estudos identificando os pontos chave para hierarquização e síntese das ideias.

Objetivando uma melhor compreensão e visualização dos principais achados organizaram-se os dados apresentando-os em figuras e tabelas, expostos de forma descritiva.

\section{RESULTADOS}

Os estudos levantados estão dispostos evidenciando seus títulos, autores, anos de publicação, níveis de evidências, objetivos e resultados. Após a leitura dos artigos selecionados, os estudos foram categorizados, classificando o conhecimento produzido sobre o tema, em níveis de evidência, majoritariamente nível VI - evidências derivadas de um único estudo descritivo ou qualitativo. Os principais achados dispostos nos objetivos e conclusões, estão diretamente associados à atuação da enfermagem na assistência ao paciente em cuidados paliativos (Tabela 1).

\begin{tabular}{|c|c|c|c|c|c|c|}
\hline N & Título/Base & $\begin{array}{c}\text { Autores } \\
\text { (Ano) }\end{array}$ & País & $\begin{array}{l}\text { Nível de } \\
\text { Evidência }\end{array}$ & Objetivo & Resultados \\
\hline 1 & $\begin{array}{l}\text { Profissionais de } \\
\text { enfermagem: com- } \\
\text { preensão sobre } \\
\text { cuidados paliativos } \\
\text { pediátricos / } \\
\text { BDENF }\end{array}$ & $\begin{array}{l}\text { Verri, Edna } \\
\text { Regina et al. } \\
(2019)(10)\end{array}$ & Brasil & VI & $\begin{array}{l}\text { Investigar a compreensão } \\
\text { e a prática dos profissio- } \\
\text { nais de enfermagem so- } \\
\text { bre os cuidados paliativos } \\
\text { pediátricos. }\end{array}$ & $\begin{array}{l}\text { Apresentaram-se, pelos profissionais, dificuldades } \\
\text { relacionadas à compreensão da filosofia e aos } \\
\text { objetivos dos cuidados paliativos e dificuldade em } \\
\text { atuar com pacientes pediátricos que estão sob esse } \\
\text { cuidado, destacando-se os sentimentos de fracasso e } \\
\text { de tristeza ao lidarem com a situação. }\end{array}$ \\
\hline
\end{tabular}

Intervenção em cuidados paliati-

2 vos: conhecimento e percepção dos enfermeiros. I BDENF
Silva,

Hashilley Alberto da et al. (2018)
Avaliar a percepção dos enfermeiros sobre cuidado paliativo antes e depois de uma intervenção.
A percepção dos enfermeiros acerca dos cuidados paliativos foi deficiente. Esse fato esteve associado à deficiência na formação técnico-científica ainda na graduação.

Para oferecer um tratamento personalizado que ofereça suporte, alívio e esperança, considerando a pessoa como um todo dentro do ambiente familiar, o enfermeiro pode utilizar a taxonomia de Nanda

classificação internacional de diagnósticos de enfermagem (Nanda-I), classificação de resultados de enfermagem (Noc) e intervenções de enfermagem classificação (Nic).
Delirium onset within a palliative nursing care for the patient and
3. care programme: family. / DOAJ
Caleffi, Dália

et al. (2018) Itália VI

(12)
Prevenir a incidência de delirium identificando pessoas em risco.
Conhecer a percepção do paciente oncológico acerca da prática dos cuidados de enfermagem, especialmente dos cuidados paliativos realizados em uma clínica cirúrgica num hospital público no oeste catarinense, sul do Brasil.

\begin{tabular}{|c|c|c|c|c|c|c|}
\hline 5 & $\begin{array}{l}\text { Vivência de } \\
\text { enfermeiros acerca } \\
\text { dos cuidados palia- } \\
\text { tivos. / LILACS }\end{array}$ & $\begin{array}{c}\text { Santos, } \\
\text { Andrea } \\
\text { Moreira dos } \\
\text { et al. (2020) } \\
\text { (14) }\end{array}$ & Brasil & VI & $\begin{array}{l}\text { Analisar a percepção de } \\
\text { enfermeiros acerca da } \\
\text { sua vivência em cuidados } \\
\text { paliativos. }\end{array}$ & $\begin{array}{l}\text { Os enfermeiros destacam que os cuidados paliativos } \\
\text { devem contemplar não só a atenção aos pacientes, } \\
\text { mas a família, ainda revelando sentimentos e medi- } \\
\text { das importantes como afeto, carinho e conforto. }\end{array}$ \\
\hline
\end{tabular}




\author{
The Experiences of \\ Family Caregivers \\ at the End of \\ Life: Suffering, \\ Compassion \\ 6 Satisfaction and \\ Ortega-Ga- \\ lan, AM et \\ al. (2019) nha \\ VI \\ Support of Health
Care Professionals \\ Experiences of \\ Caregivers at the \\ End of Life. / Web \\ (15) \\ Identificar, por meio de \\ vivências e percepções \\ de cuidadores familiares, \\ os elementos-chave \\ relacionados ao cuidado \\ oferecido ao paciente em \\ fase terminal de vida.
}

Os resultados indicam a existência de 3 dimensões: o sofrimento do cuidador, a compaixão, a satisfação com o cuidado prestado e 0 apoio dos profissionais de saúde, principalmente do enfermeiro.

\section{DISCUSSÃO}

Foi possível observar algumas dificuldades associadas a atuação da enfermagem na assistência ao paciente em cuidados paliativos, como por exemplo, alguns profissionais não conseguem compreender a filosofia de tais cuidados; o que muitas vezes está relacionado ao déficit na formação técnico-científica ainda na graduação. Ainda, é visto que há uma barreira, no que diz respeito a incentivar a implantação dos cuidados paliativos no ambiente hospitalar.

Verri et al., ${ }^{(10)}$ e Santos et al., (14) observaram em seus estudos que os profissionais de enfermagem em sua maioria, possuem dificuldades associadas ao entendimento da filosofia e os princípios dos cuidados paliativos, e ainda mais se prestados a pacientes pediátricos, acarretando sentimentos de fracasso e tristeza.

Corroborando, um estudo de Arrieira et al., ${ }^{(16)}$ constatou que o profissional, incapaz de enfrentar seus próprios problemas de morte, tem mais dificuldade em enfrentar a morte do outro e tenta de alguma forma distanciá-lo de si mesmo, o que se manifesta na fragmentação em órgãos do paciente ou por meio de sua doença ou de seus sintomas físicos.

Silva et al., (11) observaram em seu estudo a presença de um déficit acerca da percepção do enfermeiro quanto aos cuidados paliativos, este associado a uma falha na formação técnico-científica ao longo da graduação. Corroborando, Chover-Sierra, Martínez-Sabater e Lapeña-Moñux ${ }^{(17)}$ identificaram equívocos e déficits de treinamento no contexto dos cuidados paliativos entre a equipe de enfermagem. O conhecimento que seria melhorado por meio do desenvolvimento de atividades educacionais que enfocam especificamente os mal-entendidos identificados permitiria aos profissionais fornecer cuidados paliativos de maior qualidade a seus pacientes com base nas evidências científicas existentes.

Um estudo de Caleffi et al., ${ }^{(12)}$ indicam que para que seja ofertado um tratamento personalizado para o paciente, o profissional pode fazer uso da taxonomia de Nanda Classificação Internacional de Diagnósticos de Enfermagem (NANDA-I), Classificação de Resultados de Enfermagem (NOC) e Intervenções de Enfermagem Classificação (NIC).

Corroborando, Carvalho et al., (18) levanta que para fortalecer as práticas de saúde por meio do trabalho dos profissionais e das partes interessadas, recomenda-se o desenvolvimento de planos de responsabilização para uma atenção primária à saúde mais ampla e a realização do direito à saúde. Cuidados paliativos, trabalho em equipe multiprofissional e integrado a rede de saúde.
Um estudo de Ascari, Didoné e Aumondi ${ }^{(13)}$ levantou dados acerca da percepção do paciente que recebeu assistência de cuidados paliativos prestados pelo profissional de enfermagem, e estes enfatizaram o auxílio com a higiene, a troca de curativos, verificação de sinais vitais, infusões venosas, e falta de orientações sobre tais procedimentos e cuidados de enfermagem. Corroborando, um estudo de Ortega-Galan et al., ${ }^{(15)}$ afirma a existência de 3 dimensões: o sofrimento do cuidador, a compaixão, a satisfação com o cuidado prestado e o apoio dos profissionais de saúde, principalmente do enfermeiro.

Por meio das estratégias de busca utilizada encontrou-se uma amostra pequena e pouca disponibilidade de artigos científicos para comparação dos resultados. Embora tenham aparecido muitos artigos como resultado dos descritores, poucos atenderam ao objetivo do estudo. Além disso, os estudos incluídos apresentam limitações como: unicêntricos, diferentes sistemas de comparação, tamanho pequeno da amostra e falta de randomização.

Desta forma, não foi possível constatar que existe evidência científica referente a atuação da enfermagem na assistência ao paciente em cuidados paliativos. Fazendo-se necessário a realização de mais estudos contendo uma amostra maior e possibilitando discussão acerca da atuação da enfermagem na assistência ao paciente em cuidados 
paliativos

Esta pesquisa pode ajudar a disseminar o conceito e os tipos de cuidados paliativos prestados pela equipe multidisciplinar, especialmente a enfermagem. Aumentando a consciência do profissional e ampliando a visão de cuidado para com o paciente. Também, no contexto da gestão universitária, porque o ensino pode criar e disseminar o conhecimento ainda na graduação, o que pode refletir em mui- tos aspectos da prática profissional do enfermeiro.

\section{CONCLUSÃO}

Este estudo proporcionou a identificação de algumas dificuldades e das características associadas à atuação da enfermagem na assistência ao paciente em cuidados paliativos; e frequentemente vinculado a déficit de conhecimento e falta de incentivo. No entan- to, há escassez de estudos que dão a verdadeira importância a este tema, essenciais na formação, profissão, $e$ educação permanente, ainda que esse número tenha aumentado gradualmente nos últimos anos. Logo, este estudo proporcionou a percepção de que, ainda que tímido, há um crescimento no número de estudos que identificam os benefícios da atuação do enfermeiro no que condiz aos cuidados paliativos.

\section{Referências}

1. Markus LA, Betiolli SE, Souza SJP, Marques FR, Migoto MT. A atuação do enfermeiro na assistência ao paciente em cuidados paliativo. RGS, 2017; 17 (Supl 1): 71-81.

2. Santos JBS, Carvalho DMS, Fonseca MM, Silva FP. Assistência integral de enfermagem aos pacientes em cuidados paliativos. Revista Saúde, 2017; 11(1).

3. Costa BM, Silva DA. Atuação da equipe de enfermagem em cuidados paliativos. Research, Society And Development, 2021; 10 (2): e28010212553. http://doi.org/10.33448/ rsd-v10i2.12553.

4. Tomaszewski AS, Oliveira SG, Arrieira ICO, Cardosos DH, Sartor SF. Manifestações e necessidades referentes ao processo de morte e morrer: perspectiva da pessoa com câncer. Revista de Pesquisa: Cuidado é Fundamental Online, 2017; 9(3): 705-716. http://dx.doi. org/10.9789/2175- 5361.2017.v9i3.705-716

5. Picollo DP, Fachini M. A atenção do enfermeiro ao paciente em cuidado paliativo. Rev Ciênc Med. 2018;27(2):85-92. http://dx.doi. org/10.24220/2318-0897v27n2a3855

6. Ercole FF, Melo LS, Alcoforado CLGC. Integrative review versus systematic review. Reme: Revista Mineira de Enfermagem, 2014; 18 (1). GN1 Genesis Network. http://doi. org/10.5935/1415-2762.20140001.

7. Lisboa MT. Elementos para elaboração de um desenho de pesquisa | Elements to formulate a research design. Mural Internacional, 2019, 10:38439-1. Universidade de Estado do Rio de Janeiro. http://doi.org/10.12957/rmi.2019.38439.

8. Barbosa FT, Lira AB, Oliveira Neto OB, Santos LL, Santos IO, Barbosa LT et al. Tutorial for performing systematic review and meta-analysis with interventional anesthesia studies. Brazilian Journal of Anesthesiology (English Edition), 2019; 69(3): 299-306. https://doi. org/10.1016/j.bjan.2018.11.007.

9. Melnyk BM, Fineout-Overholt E. Making the case for evidence-based practice. In B. M. Melnyk \& E. Fineout-Overholt. Evidence-based practice in nursing \& healthcare: a guide to best practice. 2005; 3-24. Philadelphia: Lippincot Williams \& Wilkins.

10. Verri ER, Bitencourt NAS, Oliveira JAS, Santos Júnior R, Marques HS, Porto MA et al. Profissionais de enfermagem: compreensão sobre cuidados paliativos pediátricos.. Rev. enferm. UFPE on line., 2019; 13(1): 126-136. https://doi.org/10.5205/1981-8963-v13i01 a234924p126-136-2019
11. Silva HA, Viana GKB, Lima AKG, Lima ALA, Mourão CML. Intervenção em cuidados paliativos: conhecimento e percepção dos enfermeiros. Rev. enferm. UFPE on line, [Internet] 2018; [cited 2021 jul 19] 2(5): 1325-1330. Available from: https://pesquisa.bvsalud.org/ portal/resource/pt/biblio-980775

12. Caleffi D, Fabbo A, Gelati L, Monti C. Delirium onset within a palliative care program me: nursing care for the patient and family. Geriatr Care [Internet]. 2018 Sep. 25 [cited 2021 Jul.14];4(2). Available from: https://www.pagepressjournals.org/index.php/gc/article/ view/7563 https://doi.org/10.4081/gc.2018.7563

13. Ascari $R$, Didoné $P$, Aumondi, $C$. Percepción del paciente con cáncer sobre la práctica de los cuidados de enfermería. Cultura de los cuidados, 2018; 0(49): 14-24. https://doi. org/10.14198/cuid.2017.49.02

14. Santos AM, Narciso AC, Evangelista CB, Filgueiras TF, Costa MML, Cruz RAO. Vivência de enfermeiros acerca dos cuidados paliativos. Rev Fun Care Online. 2020 jan/dez; 12:479484. http://doi.org/10.9789/2175-5361.rpcfo.v12.8536

15. Ortega-Galán AM, Ruiz-Fernández MD, Carmona-Rega MI, Cabrera-Troya J, Ortíz-Amo $R$, Ibáñez-Masero 0. The Experiences of Family Caregivers at the End of Life: suffering, compassion satisfaction and support of health care professionals. Journal Of Hospice \& Palliative Nursing, 2019, 21 (5): 438-444. Technologies (Wolters Kluwer Health). http://doi. org/10.1097/njh.0000000000000586.

16. Arrieira ICO, Thofehrn MB, Porto, Adrize Rutz; Moura, Pedro Márlon Martter; Martins, Caroline Lemos; Jacondino, Michelle Barboza. Espiritualidade nos cuidados paliativos: experiência vivida de uma equipe interdisciplinar. Revista da Escola de Enfermagem da Usp, 2018; 52. FapUNIFESP (SciELO). http://doi.org/10.1590/s1980-220x2017007403312

17. Chover-Sierra E, Martínez-Sabater A, Lapeña-Moñux Y. Knowledge in palliative care of nursing professionals at a Spanish hospital. Revista Latino-Americana de Enfermagem, 2017; 25: e2847. https://doi.org/10.1590/1518-8345.1610.2847.

18. Carvalho GAFL, Menezes RMP, Enders BC, Teixeira GA, Dantas DNA, Oliveira DRC Significados Atribuídos Por Profissionais De Saúde Aos Cuidados Paliativos No Contexto Da Atenção Primária. Texto \& Contexto - Enfermagem, 2018; 27 (2). FapUNIFESP (SciELO). http://doi.org/10.1590/0104-070720180005740016. 